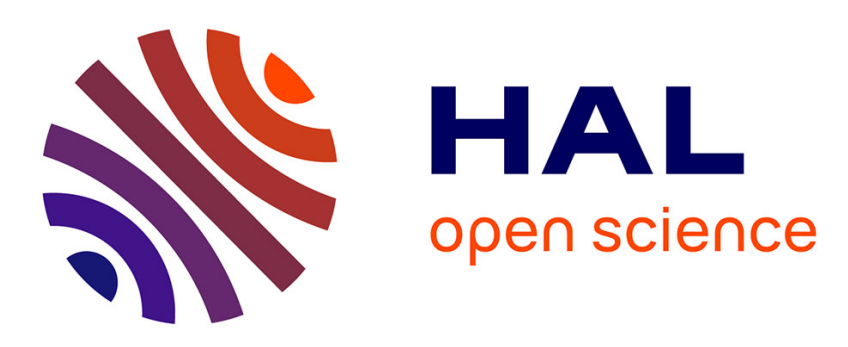

\title{
First-order characterization and modal analysis of indiscernibility and complementarity in information systems
}

Philippe Balbiani, Dimiter Vakarelov

\section{- To cite this version:}

Philippe Balbiani, Dimiter Vakarelov. First-order characterization and modal analysis of indiscernibility and complementarity in information systems. 6th European Conference on Symbolic and Quantitative Approaches to Reasoning with Uncertainty (ECSQARU 2001), Sep 2001, Toulouse, France. pp.772-781, 10.1007/3-540-44652-4_68. hal-03252571

\section{HAL Id: hal-03252571 \\ https://hal.science/hal-03252571}

Submitted on 7 Jun 2021

HAL is a multi-disciplinary open access archive for the deposit and dissemination of scientific research documents, whether they are published or not. The documents may come from teaching and research institutions in France or abroad, or from public or private research centers.
L'archive ouverte pluridisciplinaire HAL, est destinée au dépôt et à la diffusion de documents scientifiques de niveau recherche, publiés ou non, émanant des établissements d'enseignement et de recherche français ou étrangers, des laboratoires publics ou privés. 


\title{
First-order characterization and modal analysis of indiscernibility and complementarity in information systems
}

\author{
Philippe Balbiani $^{1}$ and Dimiter Vakarelov ${ }^{2}$ \\ 1 Institut de recherche en informatique de Toulouse \\ 118 route de Narbonne, 31062 Toulouse Cedex 4, France \\ ${ }^{2}$ Department of Mathematical Logic with Laboratory for Applied Logic \\ Faculty of Mathematics and Computer Science, Sofia University \\ blvd James Bouchier 5, 1126 Sofia, Bulgaria
}

\begin{abstract}
In this paper, we study indiscernibility relations and complementarity relations in information systems. The first-order characterization of indiscernibility and complementarity is obtained through a duality result between information systems and certain structures of relational type characterized by first-order conditions. The modal analysis of indiscernibility and complementarity is performed through a modal logic which modalities correspond to indiscernibility relations and complementarity relations in information systems.
\end{abstract}

\section{Introduction}

Information systems are knowledge-based systems which describe properties of objects in terms of attributes. They provide an effective and broadly applicable framework for the management and the processing of uncertainty, a crucial issue in the development of reasoning systems that are concerned with incomplete information. The increasing number of knowledge-based systems that manage and process incomplete information leads us to develop formal methods for reasoning about uncertain knowledge discovered from information systems. Initiated by Pawlak [8] and furthered by Demri [2], Demri, Orłowska and Vakarelov [3], Orłowska [5, 6], Orłowska and Pawlak [7] and Vakarelov [9, 10, 11, 12], the theoretical foundations of information systems investigate the relationships between objects determined by their properties. All the relations defined in this context are either indistinguishability relations or distinguishability relations. Indistinguishability relations indicate the way objects share properties whereas distinguishability relations indicate the way properties differentiate objects. Typical issues are the following: first-order characterization and modal analysis of various classes of indistinguishability relations and distinguishability relations. To obtain the first-order characterization of a class of indistinguishability relations and distinguishability relations, one has to find first-order conditions such that relations satisfying these conditions correspond to the indistinguishability relations and the distinguishability relations of this class derived from information systems. To perform the modal analysis of a class of indistinguishability relations 
and distinguishability relations, one has to address the questions of axiomatization/completeness and decidability/complexity of a modal logic which modalities correspond to the indistinguishability relations and the distinguishability relations of this class. In this paper, extending the line of reasoning suggested by Demri, Orłowska and Vakarelov [3], we study indiscernibility relations and complementarity relations in information systems. The first-order characterization of indiscernibility and complementarity is obtained through a duality result between certain structures of relational type characterized by first-order conditions and information systems. The modal analysis of indiscernibility and complementarity is performed through a modal logic which modalities correspond to indiscernibility relations and complementarity relations in information systems.

\section{Indiscernibility and complementarity}

Adapted from Pawlak [8], an information system will be any structure (Att, $\left.O b j,\left\{V_{a l} \mid a \in A t t\right\}, f\right)$ where:

- Att is a nonempty set of attributes;

- $O b j$ is a nonempty set of objects;

- For all $a \in A t t, V_{a l}$ is a nonempty subset of a fixed nonempty set $V a l$ of properties;

- $f$ is a function with domain $A t t \times O b j$ and range the power set of $V a l$ such that for all $a \in A t t$ and for all $x \in O b j, f(a, x) \subseteq V a l_{a}$.

We should consider, for example, the information system $S=\left(A t t, O b j,\left\{V a l_{a} \mid\right.\right.$ $a \in A t t\}, f)$ defined as follows. Define:

- Att is $\{$ Languages, Sports $\}$;

- Obj is $\{$ Ann, Bob, Cindy, Daniel, Emma\};

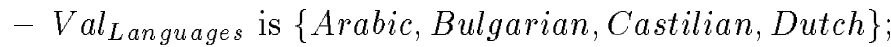

- Val Sports is $\{$ athletics, basketball, cycling\};

$-f$ is the function defined by table 1 .

In this information system, the object Bob possesses the properties Arabic and Bulgarian of mastering Arabic and Bulgarian whereas the object Daniel possesses the properties athletics and cycling of practising in athletics and cycling. Information systems constitute the starting point for the formal examination of sentences of the form "object $x$ is indistinguishable from object $y$ " or sentences of the form "object $x$ is distinguishable from object $y$ ". In this respect, indiscernibility relations and complementarity relations play an important role. Let $S=\left(A t t, O b j,\left\{V_{a l} \mid a \in A t t\right\}, f\right)$ be an information system. For all $x, y \in O b j$, define:

Strong indiscernibility: $x \equiv_{S} y$ iff for all $a \in A t t, f(a, x)=f(a, y)$;

Strong complementarity: $x R_{S} y$ iff for all $a \in A t t, f(a, x)=\left(\operatorname{Val}_{a} \backslash f(a, y)\right)$. 


\begin{tabular}{|l|l|l|l|l|l|}
\hline $\mathrm{f}$ & Ann & Bob & Cindy & Daniel & Emma \\
\hline Languages & $\begin{array}{l}\text { Arabic, } \\
\text { Bulgarian }\}\end{array}$ & $\begin{array}{l}\{\text { Arabic, } \\
\text { Bulgarian }\}\end{array}$ & $\begin{array}{l}\{\text { Castilian, } \\
\text { Dutch }\}\end{array}$ & $\begin{array}{l}\{\text { Arabic, } \\
\text { Bulgarian }\}\end{array}$ & $\begin{array}{l}\text { Arabic, } \\
\text { Castilian }\}\end{array}$ \\
\hline Sports & $\begin{array}{l}\text { athletics, } \\
\text { basketball }\}\end{array}$ & $\begin{array}{l}\{\text { athletics, } \\
\text { basketball }\}\end{array}$ & $\{$ cycling $\}$ & $\begin{array}{l}\{\text { athletics, }, \\
\text { cycling }\}\end{array}$ & $\{$ cycling $\}$ \\
\hline
\end{tabular}

Table 1. Example of an information system.

Intuitively, two objects are strongly indiscernible if all their respective sets of properties determined by the attributes are indiscernible whereas two objects are strongly complementary if all their respective sets of properties determined by the attributes are complementary. The information system of table 1 is such that $A n n \equiv_{S} B o b$ and $A n n R_{S} C i n d y$. For all $x, y \in O b j$, define:

Weak indiscernibility: $x \cong_{S} y$ iff there is $a \in$ Att such that $f(a, x)=f(a, y)$; Weak complementarity: $x \rho_{S} y$ iff there is $a \in A t t$ such that $f(a, x)=\left(V_{a l} \backslash\right.$ $f(a, y))$.

Intuitively, two objects are weakly indiscernible if some of their respective sets of properties determined by the attributes are indiscernible whereas two objects are weakly complementary if some of their respective sets of properties determined by the attributes are complementary. The information system of table 1 is such that $A n n \cong_{S}$ Daniel and Ann $\rho_{S}$ Emma. The structure $\left(O b j, \equiv_{S}, R_{S}, \cong_{S}, \rho_{S}\right)$ is called abstract structure derived from $S$. We leave it to the reader to prove the following lemmas.

Lemma 1. For all $x, y, z \in O b j$ :

$$
\begin{array}{ll}
x \equiv_{S} x ; & x \overline{R_{S}} x ; \\
\text { If } x \equiv_{S} y \text { then } y \equiv_{S} x ; & \text { If } x R_{S} y \text { then } y R_{S} x ; \\
\text { If } x \equiv_{S} y \text { and } y \equiv_{S} z \text { then } x \equiv_{S} z ; & \text { If } x R_{S} y \text { and } y \equiv_{S} z \text { then } x R_{S} z ; \\
\text { If } x \equiv_{S} y \text { and } y R_{S} z \text { then } x R_{S} z ; & \text { If } x R_{S} y \text { and } y R_{S} z \text { then } x \equiv_{S} z .
\end{array}
$$

Lemma 2. For all $x, y, z \in O b j$ :

$$
\begin{array}{ll}
x \cong_{S} x ; & x \overline{\rho_{S}} x ; \\
\text { If } x \cong_{S} y \text { then } y \cong_{S} x ; & \text { If } x \rho_{S} y \text { then } y \rho_{S} x ; \\
\text { If } x \cong_{S} y \text { and } y \equiv_{S} z \text { then } x \cong_{S} z ; & \text { If } x \rho_{S} y \text { and } y \equiv_{S} z \text { then } x \rho_{S} z ; \\
\text { If } x \cong_{S} y \text { and } y R_{S} z \text { then } x \rho_{S} z ; & \text { If } x \rho_{S} y \text { and } y R_{S} z \text { then } x \cong_{S} z
\end{array}
$$

Lemma 1 and lemma 2 motivate the following definition. An abstract structure is a structure $(W, \equiv, R, \cong, \rho)$ where:

- $W$ is a nonempty set of possible worlds;

- $\equiv$ and $R$ are binary relations on $W$ subject to the conditions of lemma 1 ;

- $\cong$ and $\rho$ are binary relations on $W$ subject to the conditions of lemma 2 . 
In section 3, the concept of abstract structure will be of use to us for the purpose of giving a first-order characterization of indiscernibility relations and complementarity relations in information systems. In section 4 , the concept of abstract structure will be of use to us for the purpose of giving a modal analysis of indiscernibility relations and complementarity relations in information systems.

\section{First-order characterization}

The concept of abstract structure is of use to us for the purpose of giving a firstorder characterization of indiscernibility relations and complementarity relations in information systems. The following important theorem explains the connection between abstract structures and information systems. All the section 3 is devoted to its proof.

Theorem 3. Let $F=(W, \equiv, R, \cong, \rho)$ be an abstract structure. There is an information system $S=\left(A t t, O b j,\left\{V a l_{a} \mid a \in A t t\right\}, f\right)$ such that $O b j=W$ and for all $x, y \in O b j$ :

$$
\begin{array}{ll}
x \equiv_{S} y \text { iff } x \equiv y ; & x \cong_{S} y \text { iff } x \cong y \\
x R_{S} y \text { iff } x R y ; & x \rho_{S} y \text { iff } x \rho y .
\end{array}
$$

As a consequence, abstract structures and information systems have equal mathematical content as far as indiscernibility relations and complementarity relations are concerned. Holding the proof of theorem 3 in abeyance for a while, we proceed to introduce the concepts of indiscernibility set, positive set, negative set and good set. Two subsets $A$ and $B$ of $W$ are called comparable if $A \subseteq B$ or $B \subseteq A$. A set of pairwise comparable subsets of $W$ is called a chain. A subset $A$ of $W$ such that for all $x, y \in W$ :

- If $x \equiv y$ and $x \in A$ then $y \in A$;

- If $x \equiv y$ and $x \notin A$ then $y \notin A$;

will be defined to be an indiscernibility set. An indiscernibility set $A$ such that for all $x, y \in W$ :

- If $x R y$ and $x \in A$ then $y \notin A$;

will be defined to be a positive set. An indiscernibility set $A$ such that for all $x, y \in W$ :

- If $x R y$ and $x \notin A$ then $y \in A$;

will be defined to be a negative set. An indiscernibility set $A$ such that $A$ is a positive set and $A$ is a negative set will be defined to be a good set. The proof of the following lemmas is left as an exercise for the reader.

Lemma 4. - $\emptyset$ and $W$ are indiscernibility sets.

- For all $x \in W, \equiv(x)$ is an indiscernibility set.

- For all $x, y \in W, \equiv(x) \cup \equiv(y)$ is an indiscernibility set. 
- For all indiscernibility sets $A,(W \backslash A)$ is an indiscernibility set.

- For all families $\left(A_{i} \mid i \in I\right)$ of indiscernibility sets, $\bigcup\left(A_{i} \mid i \in I\right)$ is an indiscernibility set and $\bigcap\left(A_{i} \mid i \in I\right)$ is an indiscernibility set.

Lemma 5. $\quad-\emptyset$ is a positive set.

- For all $x \in W, \equiv(x)$ is a positive set.

- For all $x, y \in W$, if $x \bar{R} y$ then $\equiv(x) \cup \equiv(y)$ is a positive set.

- For all positive sets $A,(W \backslash A)$ is a negative set.

- For all chains $\left(A_{i} \mid i \in I\right)$ of positive sets, $\bigcup\left(A_{i} \mid i \in I\right)$ is a positive set.

Lemma 6. $-W$ is a negative set.

- For all $x \in W, \not \equiv(x)$ is a negative set.

- For all $x, y \in W$, if $x \bar{R} y$ then $\not \equiv(x) \cap \not \equiv(y)$ is a negative set.

- For all negative sets $A,(W \backslash A)$ is a positive set.

- For all chains $\left(A_{i} \mid i \in I\right)$ of negative sets, $\bigcap\left(A_{i} \mid i \in I\right)$ is a negative set.

Lemma 7. Let $A$ be a positive set and $x \in W$ be such that $A \cup \equiv(x)$ is not a positive set. Then there is $y \in W$ such that $y \in A$ and $x R y$.

Lemma 8. Let $A$ be a negative set and $x \in W$ be such that $A \cap \not \equiv(x)$ is not a negative set. Then there is $y \in W$ such that $y \notin A$ and $x R y$.

A more important further consequence is the following lemma.

Lemma 9. Let $A$ be a positive set, $B$ be a negative set and $x \in W$ be such that $A \subseteq B$. Then $(A \cup \equiv(x)$ is a positive set and $A \cup \equiv(x) \subseteq B)$ or $(B \cap \not \equiv(x)$ is a negative set and $A \subseteq B \cap \not \equiv(x))$.

Proof. See Balbiani and Vakarelov [1] for details.

An important related result is the following proposition.

Proposition 10. Let $A$ be a positive set and $B$ be a negative set such that $A \subseteq$ $B$. Then there is a good set $C$ such that $A \subseteq C$ and $C \subseteq B$.

Proof. See Balbiani and Vakarelov [1] for details.

A set $a$ of good sets such that for all $x, y \in W$ :

- If $x \not y y$ then there is $A \in a$ such that $x \in A$ iff $y \notin A$;

- If $x \bar{\rho} y$ then there is $A \in a$ such that $x \in A$ iff $y \in A$;

will be defined to be a nice set. The following lemma is easy to check.

Lemma 11. The set of all good sets is a nice set.

Proof. See Balbiani and Vakarelov [1] for details.

A less obvious result is the following lemma. 
Lemma 12. For all $x, y \in W$ :

$-x \equiv y$ iff for all nice sets $a$ and for all $A \in a, x \in A$ iff $y \in A$;

- $x$ Ry iff for all nice sets $a$ and for all $A \in a, x \in A$ iff $y \notin A$;

$-x \cong y$ iff there is a nice set a such that for all $A \in a, x \in A$ iff $y \in A$;

- $x \rho y$ iff there is a nice set a such that for all $A \in a, x \in A$ iff $y \notin A$.

Proof. See Balbiani and Vakarelov [1] for details.

Referring to lemma 12 , we easily obtain a proof of theorem 3. Let $S=(A t t, O b j$, $\left.\left\{V_{a l} \mid a \in A t t\right\}, f\right)$ be the information system defined as follows. Define:

- Att is the set of all nice sets;

- $O b j$ is the set of all possible worlds;

- For all $a \in A t t, \mathrm{Val}_{a}$ is the set of all good sets $A$ such that $A \in a$;

- For all $a \in A t t$ and for all $x \in O b j, f(a, x)$ is the set of all good sets $A$ such that $A \in a$ and $x \in A$.

The reader may easily verify that for all $x, y \in O b j$ :

$$
\begin{array}{ll}
x \equiv_{S} y \text { iff } x \equiv y ; & x \cong_{S} y \text { iff } x \cong y ; \\
x R_{S} y \text { iff } x R y ; & x \rho_{S} y \text { iff } x \rho y .
\end{array}
$$

\section{Modal analysis}

The concept of abstract structure is of use to us for the purpose of giving a modal analysis of indiscernibility relations and complementarity relations in information systems. The reader is assumed to be familiar with the general concepts of modal logic, see Hughes and Cresswell [4] for details. Seeing that the condition $x \bar{R} x$ of lemma 1 and the condition $x \bar{\rho} x$ of lemma 2 are not modally definable, we need to introduce the concept of nonstandard abstract structure. A nonstandard abstract structure is a structure $(W, \equiv, R, \cong, \rho)$ where:

- $W$ is a nonempty set of possible worlds;

- $\equiv$ and $R$ are binary relations on $W$ subject to the conditions of lemma 1 but the condition $x \bar{R} x$;

- $\cong$ and $\rho$ are binary relations on $W$ subject to the conditions of lemma 2 but the condition $x \bar{\rho} x$.

The linguistic basis of our modal logic is the propositional calculus enlarged with the modalities $[\equiv],[R],[\cong]$ and $[\rho]$ corresponding to the indiscernibility relations and the complementarity relations in information systems. We define the set of all formulas as follows:

$$
\text { - } A::=p|\neg A|(A \vee B)|[\equiv] A|[R] A|[\cong] A|[\rho] A ;
$$

where $p$ ranges over a countably infinite set of propositional variables. The other standard connectives are defined by the usual abbreviations. In particular, $\langle\equiv\rangle A$ is $\neg[\equiv] \neg A,\langle R\rangle A$ is $\neg[R] \neg A,\langle\cong\rangle A$ is $\neg[\cong] \neg A$ and $\langle\rho\rangle A$ is $\neg[\rho] \neg A$. We follow the standard rules for omission of the parentheses. A model (respectively: a nonstandard model) is a structure $(W, \equiv, R, \cong, \rho, V)$ where: 
- $(W, \equiv, R, \cong, \rho)$ is an abstract structure (respectively: a nonstandard abstract structure);

$-V$ is a function with domain the set of all propositional variables and range the power set of $W$.

Let $M=(W, \equiv, R, \cong, \rho, V)$ be either a model or a nonstandard model. We define the relation "formula $A$ is true at possible world $x$ in $M$ ", denoted $M, x \models A$, as follows:

- $M, x \mid=p$ iff $x \in V(p)$;

- $M, x=\neg A$ iff $M, x \not \models A$;

- $M, x=A \vee B$ iff $M, x=A$ or $M, x=B$;

- $M, x=[\equiv] A$ iff for all $y \in W$, if $x \equiv y$ then $M, y=A$;

- $M, x=[R] A$ iff for all $y \in W$, if $x R y$ then $M, y=A$;

- $M, x=[\cong] A$ iff for all $y \in W$, if $x \cong y$ then $M, y=A$;

- $M, x \models[\rho] A$ iff for all $y \in W$, if $x \rho y$ then $M, y \models A$.

An alternative formulation is " $M$ satisfies formula $A$ at possible world $x$ ". The following lemma is basic.

Lemma 13. The following conditions are equivalent.

1. A is true at some possible world in some finite model;

2. A is true at some possible world in some model;

3. A is true at some possible world in some nonstandard model;

4. A is true at some possible world in some finite nonstandard model.

Proof. (1 implies 2): Obvious.

(2 implies 3$)$ : Obvious.

(3 implies 4): Let $M=(W, \equiv, R, \cong, \rho, V)$ be a nonstandard model and $M^{\prime}=$ $\left(W^{\prime}, \equiv^{\prime}, R^{\prime}, \cong{ }^{\prime}, \rho^{\prime}, V^{\prime}\right)$ be the finite nonstandard model defined as follows. Let $\Gamma_{A}$ be the smallest set of formulas containing the set $S f(A)$ of all subformulas of $A$ and such that for all formulas $B$, if $[\equiv] B \in \Gamma_{A}$ or $[R] B \in \Gamma_{A}$ or $[\cong] B \in \Gamma_{A}$ or $[\rho] B \in \Gamma_{A}$ then $[\equiv] B \in \Gamma_{A}$ and $[R] B \in \Gamma_{A}$ and $[\cong] B \in \Gamma_{A}$ and $[\rho] B \in \Gamma_{A}$. It should be remarked that $\operatorname{Card}\left(\Gamma_{A}\right)<4 \times \operatorname{Card}(S f(A))$. Let $=_{\Gamma_{A}}$ be the equivalence relation on $W$ defined as follows. For all $x, y \in W$, define:

$-x=\Gamma_{A} y$ iff for all formulas $B$, if $B \in \Gamma_{A}$ then $M, x \models B$ iff $M, y \models B$.

For all $x \in W$, the equivalence class of $x$ modulo $=_{\Gamma_{A}}$ is denoted $|x|$. The quotient set of $W$ modulo $=_{\Gamma_{A}}$ is denoted by $W_{\mid=_{\Gamma_{A}}}$. Define:

- $W^{\prime}$ is $W_{\mid=\Gamma_{A}}$;

- For all $x, y \in W,|x| \equiv^{\prime}|y|$ iff for all formulas $B$, if $[\equiv] B \in \Gamma_{A}$ then:

If $M, x=[\equiv] B$ then $M, y=[\equiv] B ; \quad$ If $M, x=[\cong] B$ then $M, y=[\cong] B$;

If $M, y=[\equiv] B$ then $M, x=[\equiv] B ; \quad$ If $M, y=[\cong] B$ then $M, x=[\cong] B$;

If $M, x=[R] B$ then $M, y=[R] B ; \quad$ If $M, x=[\rho] B$ then $M, y=[\rho] B$;

If $M, y \models[R] B$ then $M, x \models[R] B ; \quad$ If $M, y=[\rho] B$ then $M, x=[\rho] B$; 
- For all $x, y \in W,|x| R^{\prime}|y|$ iff for all formulas $B$, if $[R] B \in \Gamma_{A}$ then:

If $M, x=[\equiv] B$ then $M, y=[R] B ; \quad$ If $M, x \models[\cong] B$ then $M, y=[\rho] B$;

If $M, y=[\equiv] B$ then $M, x=[R] B ; \quad$ If $M, y=[\cong] B$ then $M, x=[\rho] B$;

If $M, x=[R] B$ then $M, y=[\equiv] B ; \quad$ If $M, x=[\rho] B$ then $M, y=[\cong] B$;

If $M, y=[R] B$ then $M, x=[\equiv] B ; \quad$ If $M, y=[\rho] B$ then $M, x=[\cong] B$;

- For all $x, y \in W,|x| \cong \cong^{\prime}|y|$ iff for all formulas $B$, if $[\cong] B \in \Gamma_{A}$ then:

If $M, x=[\cong] B$ then $M, y=[\equiv] B ; \quad$ If $M, x \models[\rho] B$ then $M, y=[R] B$;

If $M, y=[\cong] B$ then $M, x=[\equiv] B ; \quad$ If $M, y=[\rho] B$ then $M, x=[R] B$;

- For all $x, y \in W,|x| \rho^{\prime}|y|$ iff for all formulas $B$, if $[\rho] B \in \Gamma_{A}$ then:

If $M, x=[\cong] B$ then $M, y=[R] B ; \quad$ If $M, x=[\rho] B$ then $M, y=[\equiv] B$; If $M, y=[\cong] B$ then $M, x=[R] B ; \quad$ If $M, y=[\rho] B$ then $M, x=[\equiv] B$;

- For all propositional variables $p, V^{\prime}(p)$ is $V(p)_{\mid=_{\Gamma_{A}}}$.

It follows immediately that $M^{\prime}$ is a filtration of $M$. As a consequence, if $A$ is true at some possible world in $M$ then $A$ is true at some possible world in $M^{\prime}$. (4 implies 1): Let $M=(W, \equiv, R, \cong, \rho, V)$ be a finite nonstandard model and $M^{\prime}=\left(W^{\prime}, \equiv^{\prime}, R^{\prime}, \cong^{\prime}, \rho^{\prime}, V^{\prime}\right)$ be the finite model defined as follows. Define:

- $W^{\prime}$ is $W \times\{0,1\}$;

- For all $x, y \in W$ and for all $i, j \in\{0,1\},(x, i) \equiv^{\prime}(y, j)$ iff $x \equiv y$ and $i=j$;

- For all $x, y \in W$ and for all $i, j \in\{0,1\},(x, i) R^{\prime}(y, j)$ iff $x R y$ and $i=1-j$;

- For all $x, y \in W$ and for all $i, j \in\{0,1\},(x, i) \cong(y, j)$ iff $x \cong y$ and $i=j$;

- For all $x, y \in W$ and for all $i, j \in\{0,1\},(x, i) \rho^{\prime}(y, j)$ iff $x \rho y$ and $i=1-j$;

- For all propositional variables $p, V^{\prime}(p)$ is $V(p) \times\{0,1\}$.

It follows immediately that $M$ is a p-morphic image of $M^{\prime}$. As a consequence, if $A$ is true at some possible world in $M$ then $A$ is true at some possible world in $M^{\prime}$.

Now we turn to the axiomatization of the set of all formulas true at all possible worlds in all models. Let $L S W I C$ - logic of strong and weak indiscernibility and complementarity - be the smallest normal modal logic that contains the axioms of table 2. A typical result is the following.

Theorem 14. LSWIC is complete with respect to the class of all models and the class of all nonstandard models, i.e. the following conditions are equivalent.

1. A is true at all possible worlds in all models;

2. $A$ is true at all possible worlds in all nonstandard models;

3. $A$ is a theorem of $L S W I C$.

Proof. (1 implies 2): By lemma 13.

(2 implies 1): By lemma 13.

(2 implies 3): The proof can be obtained by the canonical model construction.

(3 implies 2): The proof is trivial because nonstandard models satisfy the conditions which are needed to verify the axioms of $L S W I C$. 


$$
\begin{array}{ll}
{[\equiv] A \rightarrow A} & {[\cong] A \rightarrow A} \\
A \rightarrow[\equiv]\langle\equiv\rangle A & A \rightarrow[\cong]\langle\cong\rangle \\
{[\equiv] A \rightarrow[\equiv][\equiv] A} & {[\cong] A \rightarrow[\cong][\equiv] A} \\
{[R] A \rightarrow[\equiv][R] A} & {[\rho] A \rightarrow[\cong][R] A} \\
A \rightarrow[R]\langle R\rangle A & A \rightarrow[\rho]\langle\rho\rangle A \\
{[R] A \rightarrow[R][\equiv] A} & {[\rho] A \rightarrow[\rho][\equiv] A} \\
{[\equiv] A \rightarrow[R][R] A} & {[\cong] A \rightarrow[\rho][R] A}
\end{array}
$$

Table 2. Axioms of $L S W I C$.

We now turn our attention to the decidability of the problem of determining of any given formula whether it is a theorem of $L S W I C$ or not.

Theorem 15. Determining of any given formula whether it is a theorem of $L S W I C$ or not is decidable.

Proof. By lemma 13 and theorem 14, LSWIC is a finitely axiomatizable normal modal logic which has the finite model property. As a consequence, determining of any given formula whether it is a theorem of $L S W I C$ or not is decidable.

\section{Conclusion}

We have addressed the issues of first-order characterization and modal analysis of indiscernibility and complementarity in information systems. Previous first-order characterizations and modal analyses have been given by Demri [2], Demri, Orłowska and Vakarelov [3], Orłowska [5, 6], Orłowska and Pawlak [7] and Vakarelov [9, 10, 11, 12] who consider indistinguishability relations and distinguishability relations like the similarity relations defined as follows. Let $S=\left(A t t, O b j,\left\{V a l_{a} \mid a \in A t t\right\}, f\right)$ be an information system. For all $x, y \in O b j$, define:

Strong positive similarity: $x \sigma_{S} y$ iff for all $a \in A t t, f(a, x) \cap f(a, y) \neq \emptyset$;

Strong negative similarity: $x \nu_{S} y$ iff for all $a \in A t t,\left(\operatorname{Val}_{a} \backslash f(a, x)\right) \cap\left(\operatorname{Val}_{a} \backslash\right.$ $f(a, y)) \neq \emptyset$;

Weak positive similarity: $x \Sigma_{S} y$ iff there is $a \in$ Att such that $f(a, x) \cap f(a, y)$ $\neq \emptyset$

Weak negative similarity: $x N_{S} y$ iff there is $a \in A t t$ such that $\left(\operatorname{Val}_{a} \backslash f(a, x)\right)$ $\cap\left(\operatorname{Val}_{a} \backslash f(a, y)\right) \neq \emptyset$

It should be remarked that the strong complementarity relation is definable by means of the strong similarity relations as follows:

$-R_{S}=\overline{\sigma_{S}} \cap \overline{\nu_{S}}$ 
whereas the weak complementarity relation is definable neither by means of the strong similarity relations nor by means of the weak similarity relations. First-order characterizations and modal analyses of indiscernibility relations and complementarity relations in information systems together with other indistinguishability relations or distinguishability relations like similarity relations are not known.

\section{References}

1. Balbiani, P., Vakarelov, D.: A modal logic for indiscernibility and complementarity in information systems. To appear.

2. Demri, S.: The nondeterministic information logic NIL is PSPACE-complete. Fundamenta Informaticæ42 (2000) 211-234.

3. Demri, S., Orłowska, E., Vakarelov, D.: Indiscernibility and complementarity relations in information systems. In Gerbrandy, J., Marx, M., de Rijke, M., Venema, Y. (Editors): JFAK: Essays Dedicated to Johan van Benthem on the Occasion of his 50th Birthday. Amsterdam University Press (1999) http://turing.wins.uva.nl/ j50/cdrom/contribs/demri/index.html.

4. Hughes, G., Cresswell, M.: A Companion to Modal Logic. Methuen (1984).

5. Orłowska, E.: Logic of nondeterministic information. Studia Logica 44 (1985) 91100.

6. Orłowska, E.: Kripke semantics for knowledge representation logics. Studia Logica 49 (1990) 255-272.

7. Orłowska, E., Pawlak, Z.: Representation of nondeterministic information. Theoretical Computer Science 29 (1984) 27-39.

8. Pawlak, Z.: Information systems - theoretical foundations. Information Systems 6 (1981) 205-218.

9. Vakarelov, D.: A modal logic for similarity relations in Pawlak knowledge representation systems. Fundamenta Informaticæ15 (1991) 61-79.

10. Vakarelov, D.: Modal logics for knowledge representation systems. Theoretical Computer Science 90 (1991) 433-456.

11. Vakarelov, D.: A duality between Pawlak's knowledge representation systems and BI-consequence systems. Studia Logica 55 (1995) 205-228.

12. Vakarelov, D.: Information systems, similarity relations and modal logics. In Orłowska, E. (Editor): Incomplete Information: Rough Set Analysis. Physica-Verlag, Studies in Fuzziness and Soft Computing 13 (1998) 492-550.

This article was processed using the $\mathrm{HT}_{\mathrm{E}} \mathrm{X}$ macro package with LLNCS style 